\title{
COVID-19 ASSOCIADA A PANCREATITE AGUDA: UM RELATO DE CASO
}

\section{COVID-19 ASSOCIATED WITH ACUTE PANCREATITIS: A CASE REPORT}

Autores: Letícia Martins Paravidino', Heitor Carvalho Moulin Valencia², Letícia Oliveira Barreto', Maria Luiza Fagundes Wekid', Miguel Ribeiro Almeida', Márcia Maria Moulin Valencia ${ }^{3}$

1- Acadêmico(a) da Faculdade de Medicina de Campos

2- Acadêmico do Centro Universitário Arthur Sá Earp Neto/ Faculdade de Medicina de Petrópolis

3. Médica; Formada na Faculdade de Medicina de Campos, Atuante no Hospital Ferreira Machado e Unimed Campos

RESUMO:

A infecção pelo vírus SARS-CoV-2, comumente chamado de "novo coronavírus", foi a responsável pelo estado de pandemia declarado pela OMS em março de 2020. A COVID-19, como é popularmente conhecida, acomete principalmente as vias respiratórias superiores e inferiores. As manifestações clínicas mais comuns são febre, tosse, mialgia e fadiga. O paciente também pode apresentar secreção respiratória, dor de cabeça, hemoptise e distúrbios gastrointestinais como diarreia, náuseas, vômitos e hiporexia. Apesar da taxa de mortalidade da infecção viral não ser elevada na grande maioria da população, os indivíduos idosos, imunodeprimidos, cardiopatas, obesos, diabéticos e hipertensos, constituem o grupo de risco para a infecção pelo coronavírus. No presente estudo, relatamos o caso de uma paciente do sexo feminino, 51 anos, que apresentava como sintoma principal a dor abdominal em barra, característica de um quadro de pancreatite. Durante a internação, a paciente apresentou novos sinais e sintomas, testando positivo para COVID-19. A paciente teve alta após um longo período de internação, sinalizando a importância da equipe multidisciplinar no tratamento precoce dos indivíduos infectados peloSARS-CoV-2.

Palavras-chave: Coronavírus; Pancreatite, COVID-19, SARS-CoV-2

\section{ABSTRACT:}

The infection by the virus SARS-CoV-2, commonly known as "the new coronavirus", was responsible for the pandemic status declared by the World Health Organization (WHO) in march 2020. The COVID-19, how it's commonly named, affects mostly the respiratory system upper and lower airways. The most common clinical manifestations are fever, cough, myalgia and fatigue. The patient can also present respiratory secretions, headache, hemoptysis and gastrointestinal disorders such as diarrhea, nausea, vomiting and hyporexia. Despite the low mortality rate of the vírus in the majority of the population, a group of people that includes elderly citizens, imunodepressed, cariopaths, obese, diabetics and hypertensive patients are known as the risk group for this disease. In this current study, we report a case of a 51 year-old female patient that presented severe abdominal pain in the upper abdomen, which is a sign of pancreatitis. During hospitalization, the patient presented new signs and symptoms, with positive test results for COVID-19. The patient was discharged from the hospital after a very long hospitalization period, demonstrating the importance of a multidisciplinary team on the early treatment of the SARS-CoV-2 infected patients.

Keywords: Coronavirus; Pancreatitis; COVID-19; SARS-CoV-2 


\section{INTRODUÇÃO}

O ano de 2019 pode ser considerado um marco na história da humanidade, devido ao surto da Síndrome Respiratória Aguda Grave (SARS), iniciado na China. O vírus causador da mais recente pandemia veio a ser chamado de "novo coronavírus" o SARSCoV-2, popularmente denominada: A Pandemia do Novo Coronavírus².

Os coronavírus, da família Coronaviridae, são de vários gêneros, espécies e cepas, presentes no cotidiano, alguns infectando animais e outros infectando humanos. As afecções causadas pelo coronavírus causam, desde doenças leves com sintomas de resfriado comum, até doenças graves como a SARS, a Síndrome Respiratória no Oriente Médio (MERS) e a atual doença chamada COVID-19. As mencionadas patologias acometem principalmente as vias respiratórias (superiores e inferiores), podendo, também, provocar manifestações gastrointestinais como náuseas, vômitos, diarreia e dor/desconforto abdominal'.

A SARS se espalhou por vários países a partir da China entre 2002 e 2003, e apresentou letalidade de aproximadamente $10 \%$. Já a MERS, foi uma enfermidade oriunda de uma cepa do coronavírus, que teria surgido na Arábia Saudita no ano de 2012, disseminando-se por todo o Oriente Médio, atingindo Ásia, Europa, África e América. A estimativa de sua letalidade aproxima-se de $30 \%{ }^{9}$.

A COVID-19 é uma doença causada pelo SARS-COV-2, vírus semelhante ao que teria causado a SARS no passado, com letalidade variando no Brasil em torno de $3,0 \%{ }^{10}$, um índice relativamente baixo, que se altera de acordo com as características demográficas e de registro de cada país. Entretanto, seu potencial destrutivo se associa à grande capacidade de disseminação, inclusive através de indivíduos portadores assintomáticos, o que culmina em altos números absolutos de mortes, principalmente em indivíduos comórbidos, como: hipertensos, imunodeprimidos, diabéticos, obesos e cardiopatas. Cabe ressaltar a instalação de sequelas, que embora não muito elucidadas, já se mostraram presentes em alguns casos de recuperação da doença.

A seguir relata-se um caso de associação entre COVID-19 e pancreatite aguda, a qual é caracterizada como uma síndrome de resposta inflamatória sistêmica que tem origem pancreática, tendo como causas mais comuns cálculos biliares e abuso de álcool. Pode também ser induzida por infecções virais ou parasitárias, e o seu principal sintoma é a dor abdominal em barra ${ }^{3}$. Como alterações laboratoriais, observa-se alterações nas enzimas amilase e lipase, além de leucocitose e hiperglicemia moderada em consequência a resposta inflamatória sistêmica.

\section{OBJETIVO:}

Relatar o caso de uma paciente portadora de pancreatite aguda que contraiu COVID-19 durante seu período de internação.

\section{MÉTODOS:}

As informações foram obtidas através da revisão do prontuário, entrevista com a paciente e revisão da literatura.

\section{RELATO DE CASO}

Paciente do sexo feminino, 51 anos, hipertensa e diabética, deu entrada na emergência no dia 26/05/2020 com queixa de dor abdominal de forte intensidade em barra e vômitos incoercíveis biliosos há 5 dias. Nega diarreia e febre. Ao exame, paciente apresentava-se hipocorada 1+/4+, desidratada, PA: $110 \times 60 \mathrm{mmHg}, \mathrm{FC}: 88 \mathrm{bpm}, \mathrm{HGT}$ : 225 e restante do exame físico sem alterações, exceto, abdome doloroso à palpação superficial, porém sem sinais de irritação peritoneal. Prontamente, foi submetida a uma TC de abdome sem o uso do contraste oral ou venoso, que evidenciou pâncreas de dimensões aumentadas, contornos irregulares associados a aumento da densidade de planos gordurosos peripancreáticos e pequena coleção líquida peripancreática, sugerindo pancreatite aguda. A paciente foi avaliada pela cirurgia geral e definida como sem critérios cirúrgicos no momento. Nesta mesma data, exames séricos laboratoriais apresentavam: lipase de $141 \mathrm{U} / \mathrm{L}$; Amilase $36 \mathrm{U} / \mathrm{L}$; TGP $10 \mathrm{U} / \mathrm{Le}$ triglicerídeo de 460 $\mathrm{mg} / \mathrm{dl}$. No dia 03/06 foi realizada uma nova TC de tórax e abdome e nesta visualizou-se consolidação em lobo inferior esquerdo e pequeno derrame pleural. Em nível de abdome, infiltração e borramento da gordura peripancreática, espessamento da fáscia pararrenal anterior e uma coleção medindo $9 \mathrm{~cm}$ em seu maior diâmetro. Devido a piora do quadro e persistência dos vômitos e da dor abdominal por mais de 7 dias, a paciente foi transferida para a UTI. No dia 09/06 paciente iniciou quadro gripal, levantando suspeita de COVID-19 devido a conjuntura epidemiológica atual. Foi realizado Reação em Cadeia de Polimerase em Tempo Real (RT-PCR) 09/06 confirmando a suspeita 
previamente aventada. Frente a gasometria apresentada pela paciente e seu esforço respiratório (FR=36 irpm) optou-se pela intubação orotraqueal na tentativa de melhorar sua oxigenação. Gasometria: $\mathrm{PH}: 7,29, \mathrm{PCO} 2: 34, \mathrm{HCO}: 17, \mathrm{BE}:-9$, sat02:80\%. Iniciou-se dieta parenteral, drogas vasoativas e protocolo para COVID-19. Feito Clexane, 5 dias de Azitromicina, 5 dias de Metilprednisolona, 5 dias de Hidroxicloroquina e dose única de Ivermectina. Durante o período sintomático por COVID-19, a tomografia mostrava espessamento intra e interlobulares e opacidade em vidro-fosco bilaterais compatíveis com o quadro clínico. Houve piora progressiva do quadro, evoluindo para choque séptico e degradação da função renal. Nesse momento, foi optado por iniciar a terapia dialítica e antibioticoterapia com Meropenem, Vancomicina, Fluconazol e Polimixina B. Segue abaixo a evolução dos parâmetros gasométricos e a evolução laboratorial da pancreatite:

Evolução gasométrica da paciente (Tabela 1)

\begin{tabular}{|c|c|c|c|}
\hline $\mathrm{pH}$ & $\mathrm{pCO} 2$ & $\mathrm{HCO}-$ & Base Excess \\
\hline 7,29 & 34 & 17 & -9 \\
\hline 7,14 & 50 & 15,3 & -11 \\
\hline 7,2 & 38 & 15 & -11 \\
\hline 7,12 & 40 & 12 & -15 \\
\hline
\end{tabular}

Evolução Laboratorial da paciente (Tabela 2)

\begin{tabular}{|c|c|c|c|c|c|c|c|c|}
\hline Lipase & Amilase & \begin{tabular}{|l|l} 
Fosfatase Alcalina \\
\end{tabular} & \begin{tabular}{|l|} 
Triglicerídeos \\
\end{tabular} & Gama GT & LDH & $\mathrm{\alpha K}$ & Ureia & Creatinina \\
\hline $73 \mathrm{U} / \mathrm{L}$ & $48 \mathrm{U} / \mathrm{L}$ & - & - & - & - & - & - & - \\
\hline $60 \mathrm{U} / \mathrm{L}$ & $53 \mathrm{U} / \mathrm{L}$ & $30 \mathrm{U} / \mathrm{L}$ & $460 \mathrm{mg} / \mathrm{dl}$ & - & - & $44 \mathrm{U} / \mathrm{L}$ & - & - \\
\hline $73 \mathrm{U} / \mathrm{L}$ & $43 \mathrm{U} / \mathrm{L}$ & - & -- & $115 \mathrm{U} / \mathrm{L}$ & 199U/L & $45 \mathrm{U} / \mathrm{L}$ & - & - \\
\hline $54 \mathrm{U} / \mathrm{L}$ & $44 \mathrm{U} / \mathrm{L}$ & $26 \mathrm{U} / \mathrm{L}$ & $338 \mathrm{mg} / \mathrm{dl}$ & $137 \mathrm{U} / \mathrm{L}$ & - & - & - & - \\
\hline $45 \mathrm{U} / \mathrm{L}$ & $39 \mathrm{U} / \mathrm{L}$ & $26 \mathrm{U} / \mathrm{L}$ & - & $126 \mathrm{U} / \mathrm{L}$ & $212 \mathrm{U} / \mathrm{L}$ & - & - & - \\
\hline $76 \mathrm{U} / \mathrm{L}$ & - & $27 \mathrm{U} / \mathrm{L}$ & - & $149 \mathrm{U} / \mathrm{L}$ & $249 \mathrm{U} / \mathrm{L}$ & - & - & - \\
\hline- & - & - & - & $177 \mathrm{U} / \mathrm{L}$ & $315 \mathrm{U} / \mathrm{L}$ & - & - & - \\
\hline- & $80 \mathrm{U} / \mathrm{L}$ & $86 \mathrm{U} / \mathrm{L}$ & $685 \mathrm{mg} / \mathrm{dl}$ & $232 \mathrm{U} / \mathrm{L}$ & - & - & $126 \mathrm{~m} / \mathrm{dl}$ & 3,7 mg/dl \\
\hline- & -- & - & - & -- & - & - & $179 \mathrm{mg} / \mathrm{dL}$ & $4,8 \mathrm{mg} / \mathrm{dl}$ \\
\hline- & -- & - & - & - & - & - & $210 \mathrm{mg} / \mathrm{dL}$ & $6 \mathrm{mg} / \mathrm{dl}$ \\
\hline $133 \mathrm{U} / \mathrm{L}$ & $87 \mathrm{U} / \mathrm{L}$ & $97 \mathrm{U} / \mathrm{L}$ & $268 \mathrm{mg} / \mathrm{dl}$ & $352 \mathrm{U} / \mathrm{L}$ & - & $175 \mathrm{U} / \mathrm{L}$ & - & - \\
\hline $185 \mathrm{U} / \mathrm{L}$ & $121 \mathrm{U} / \mathrm{L}$ & - & - & $210 \mathrm{U} / \mathrm{L}$ & $679 \mathrm{U} / \mathrm{L}$ & - & - & - \\
\hline $112 \mathrm{U} / \mathrm{L}$ & $133 \mathrm{U} / \mathrm{L}$ & $60 \mathrm{U} / \mathrm{L}$ & $112 \mathrm{mg} / \mathrm{dl}$ & $370 \mathrm{U} / \mathrm{L}$ & $695 \mathrm{U} / \mathrm{L}$ & - & - & - \\
\hline
\end{tabular}

Paciente no dia 03/07 foi traqueostomizada. Neste momento apresentava-se estável do ponto de vista hemodinâmico, sem antibióticos e drogas vasoativas, porém ainda dependente da ventilação mecânica. No dia 04/08 paciente recebeu alta da UTI traqueostomizada com cânula metálica, porém com resolução completa do COVID-19 e da pancreatite.

A seguir, a linha do tempo com os eventos marcantes passados pela paciente. (Figura 1)

\section{DISCUSSÃO:}

A infecção por SARS-CoV-2apresentada pela paciente do caso, ora relatado, chama atenção para o envolvimento do sistema respiratório, além de outras

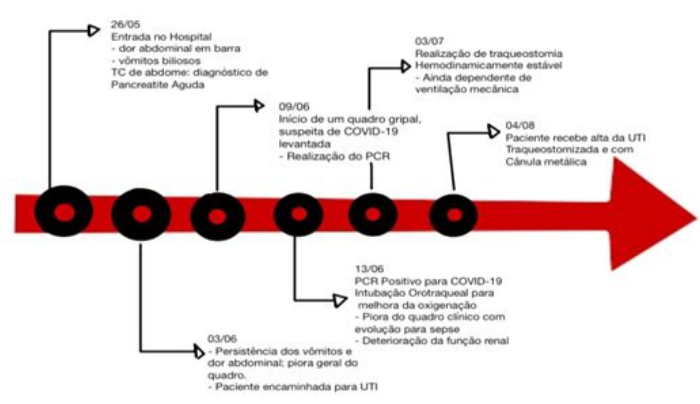

Figura 1: Linha do tempo com os eventos marcantes passados pela paciente.

apresentações complexas que se devem ao quadro de pancreatite aguda inicialmente identificado, e do envolvimento de vários sistemas, podendo ser letal se não for diagnosticado e tratado de forma adequada.

Quanto ao diagnóstico da infecção por SARSCoV-2, que no caso relatado foi feito devido à suspeita levantada a partir de um quadro gripal desenvolvido pela paciente durante o período de internação hospitalar, deve ser feito a partir da análise do material de amostragem nasofaríngea e orofaríngea, preferencialmente pelas vias aéreas inferiores (quando disponíveis). Tal análise é feita a partir de testes de RT-PCR ${ }^{6}$.

Além do RT-PCR, a determinação de anticorpos anti-SARS-CoV-2 IgM e IgG podem ser úteis na confirmação diagnóstica, sendo $\operatorname{lgM}$ detectável entre 7-10 dias a partir do início dos sintomas e IgG não detectável antes de 12 dias do início dos sintomas ${ }^{6}$.

O processo adequado para o diagnóstico se faz necessário, e uma vez que o diagnóstico seja confirmado, caso seja concomitante à outras patologias, deve-se atentar para interações medicamentosas entre os antivirais e outras classes de medicamentos. Também é necessário se atentar para a profilaxia farmacológica e não farmacológica (mobilização e hidratação) do tromboembolismo venoso, mesmo em pacientes ambulatoriais ou de alta hospitalar, caso estes tenham fator de risco adicional $^{6}$.

Quanto a saúde do profissional e de toda a equipe que presta assistência direta ao paciente, deve-se utilizar das precauções de biossegurança no manuseio de dispositivos de oxigenoterapia, manter distância de 2 metros do paciente quando possível, restringir a equipe que tem contato com o paciente infectado pelo SARS-CoV-2 para o menor número 
possível de profissionais que consigam garantir o melhor cuidado ao paciente e utilizar Equipamento de Proteção Individual (EPI) em todos os momentos ${ }^{7}$.

\section{CONCLUSÃO:}

No cenário atual onde todos estão preocupados com as consequências da pandemia da COVID-19, precisamos estar atentos e alertar a população de profissionais de saúde sobre os riscos de contaminação pelo SARS-CoV-2 em pacientes hospitalizados. Ademais, é válido ressaltar a extrema importância do trabalho de uma equipe multidisciplinar no tratamento de um paciente contaminado pelo novo coronavírus, bem como, a importância de um diagnóstico ainda na fase inicial. A infecção pelo coronavírus pode se apresentar de forma associada ou subsequente a inúmeras outras patologias, evidenciando a importância da atenção ao comportamento clínico da doença e identificação dos sinais e sintomas de forma precoce.

\section{REFERÊNCIAS}

1) Cheung K.S., Hung I.F., Chan P.P., Lung K.C., Tso E., Liu R. Gastrointestinal manifestations of SARS-CoV-2 infection and vírus load in fecal samples from the Hong Kong cohort and systematic review and meta-analysis Gastroenterology (2020), 10.1053/j.gastro.2020.03.065

2) Chih-Cheng Lai, Tzu-Ping Shih, Wen-Chien Ko, Hung-Jen Tang, Po-Ren Hsueh. Severe acute respiratory syndrome coronavirus 2 (SARS-CoV-2) and coronavirus disease-2019 (COVID-19): The epidemic and the challenges. International Journal of Antimicrobial Agents, Volume 55, Issue 3, 2020,105924, ISSN 0924-8579. Disponível em: <https://www.sciencedirect.com/science/article/pii/S0924857920300674>. Acesso em 16 set. 2020. 3) JAMESON, J. L. et al. Medicina interna de Harrison. 20. ed. Porto Alegre: AMGH, 2016. 2 v. p 2090-2102. 4) GUIMARÃES-FILHO, Marco Antônio. Pancreatite aguda: etiologia, apresentação clínica e tratamento. Revista Hospital Universitário Pedro Ernesto. Pancreatite Aguda: Etiologia, Apresentação Clínica e Tratamento, [S.I.], v. 8, n. 1, abr. 2014. ISSN 1983-2567. Disponível em: <https://www.e-publicacoes.uerj.br/index.php/revistahupe/article/view/9234>. Acesso em: 18 set. 2020.

5) Lima, FLO., Gomes, LNL., Santos, CSC dos., \& Oliveira, GAL de. (2020). Diagnóstico de COVID-19: importância dos exames laboratoriais e de imagem. Pesquisa, Sociedade e Desenvolvimento, 9 (9), e259997162. <Https://doi.org/10.33448/rsdv9i9.7162>

6) LANA, Raquel Martins. Emergência do novo coronavírus (SARS-CoV-2) e o papel de uma vigilância nacional em saúde oportuna e efetiva. Cad. Saúde Pública, Rio de Janeiro, v. 36, n. 3, e00019620, fev. 2020. Disponível em: <http://cadernos.ensp.fiocruz.br/csp/artigo/999/emergencia-do-novo-coronavirus-sars-cov-2-e-o-papel-de-uma-vigilancianacional-em-saude-oportuna-e-efetiva>. Acesso em 16 set. 2020.

7) MINISTÉRIO DA SAÚDE, BRASIL. O que é COVID-19. Brasília, DF, Ministério daSaúde,2020. Disponível em: <https://coronavirus.saude.gov.br/index.php/sobre-a-doenca>. Acesso em 16 set 2020.

8) MINISTÉRIO DA SAÚDE, BRASIL. Painel de casos de doença pelo coronavírus 2019 (COVID-19) no Brasil. (Atualizado em 18 set. 2020). BRASILIA,DF: Ministério da Saúde, 2020. Disponível em: <https://covid.saude.gov.br>. Acesso em 18 set. 2020.

9) Universidade Estadual de Campinas Unicamp. Hospital de Clínicas -HC. Manejo do paciente internado na enfermaria com COVID-19. Protocolo institucional. São Paulo. 2020. Disponível em:

$<$ https://www.fcm.unicamp.br/covid/sites/default/files/2020-

06/VERSAO\%20GOOGLEDRIVE\%20Protocolo\%20enfermaria\%20COVID\%2019-8.pdf.pdf>. Acesso em 15 de set. 2020.

10) Xavier, Ana Lucia R., Silva, Jonadab S., Almeida, João Paulo C. L., Conceição, Johnatan Felipe F., Lacerda, Gilmar S., \& Kanaan, Salim. (2020). COVID-19: manifestações clínicas e laboratoriais na infecção pelo novo coronavírus. Jornal Brasileiro de Patologia e Medicina Laboratorial, 56, e3232020. Epub 01 de julho de 2020. Disponível em:

<https://dx.doi.org/10.5935/1676-2444.20200049>. Acesso em 18 set. 2020. 